\title{
Alternate Gradient Focusing and Deceleration of a Molecular Beam
}

\author{
Hendrick L. Bethlem, André J. A. van Roij, Rienk T. Jongma, and Gerard Meijer \\ FOM-Institute for Plasma Physics Rijnhuizen, P.O. Box 1207, NL-3430 BE Nieuwegein, The Netherlands \\ and Department of Molecular and Laser Physics, University of Nijmegen, Toernooiveld 1, NL-6525 ED Nijmegen, The Netherlands
} (Received 29 October 2001; published 18 March 2002)

\begin{abstract}
Neutral dipolar molecules can be decelerated and trapped using time-varying inhomogeneous electric fields. This has been demonstrated only for molecules in low-field seeking states, but can, in principle, be performed on molecules in high-field seeking states as well. Transverse stability is then much more difficult to obtain, however, since molecules in high-field seeking states always experience a force towards the electrodes. Here we demonstrate that an array of dipole lenses in alternate gradient configuration can be used to maintain transverse stability. A pulsed beam of metastable $\mathrm{CO}$ in high-field seeking states is accelerated from 275 to $289 \mathrm{~m} / \mathrm{s}$ as well as decelerated from 275 to $260 \mathrm{~m} / \mathrm{s}$.
\end{abstract}

DOI: 10.1103/PhysRevLett.88.133003

It is now widely acknowledged that samples of cold molecules hold great promise for fundamental physics studies, and a variety of methods are currently being developed to produce such samples [1-3]. One of the methods that has recently been successful in trapping molecules [4,5] makes use of time-varying electric fields to decelerate pulsed beams of polar molecules [6,7]. This method is based on the notion that a molecule in a quantum state in which the electric dipole moment is antiparallel to an external electric field will be attracted to regions of low electric field. Therefore, molecules in this so-called "low-field seeking" state, will be decelerated on their way from a region of low electric field into a region of high electric field. If the electric field is switched off while the molecules are still in the region of high electric field, the molecules will not regain their lost kinetic energy. This process can be repeated until the average velocity is reduced to an arbitrarily low value. A properly timed switching of the electric fields ensures that a bunch of molecules can be kept together in the forward direction ("phase stability") throughout this deceleration process. Transverse stability is achieved by using an electrode geometry that produces a minimum of the electric field on the molecular beam axis, thereby continuously focusing the beam $[6,7]$.

The rotational ground state of any molecule is always lowered in energy by an external perturbation, and is therefore a "high-field seeking" state. It would be a major advantage if molecules (and atoms [8]) in this state could be decelerated as well. It might appear to be straightforward to apply the above method to molecules in high-field seeking states by simply letting the molecules fly out of, instead of into, the region of a high electric field. For the motion of the molecules in the forward direction, this is true indeed. However, Maxwell's equations do not allow for a maximum of the electric field in free space [9], e.g., on the molecular beam axis, and therefore transverse stability cannot be maintained easily; molecules in high-field seeking states have a tendency to crash into the electrodes, where the electric fields are the highest.
PACS numbers: 33.80.Ps, 33.55.Be, 39.10.+j

The same situation is encountered in charged particle accelerators, where this problem has been resolved by applying the alternate gradient (AG) focusing method [10]. This method came out of the realization that a longestablished fact of geometrical optics was applicable to ion optics as well; for a pair of lenses that have equal focal lengths but with one lens converging and the other diverging, the total focal length is always positive. This same principle can be applied to polar molecules when using electrostatic dipole lenses. These lenses focus the molecular beam in one direction but simultaneously defocus the beam in the orthogonal direction. By alternating the orientation of these lenses, an electric field geometry with a focusing effect in both directions can be created. By switching these lenses on and off at the appropriate times, AG focusing and deceleration of a molecular beam can be achieved simultaneously.

The application of AG focusing to polar molecules was first considered by Auerbach et al. [11] in a design study for a neutral particle accelerator at the University of Chicago [12]. AG focusing was experimentally demonstrated by Kakati and Lainé [13] and by Günther and co-workers [14]. Although the Chicago group demonstrated AG focusing of a molecular beam as well, their attempts to demonstrate acceleration remained without success [15]. More recently, AG focusing has been used to image an atomic beam [16]. In this Letter we demonstrate acceleration and deceleration of polar molecules in high-field seeking states. A pulsed beam of metastable CO is accelerated from 275 to $289 \mathrm{~m} / \mathrm{s}$ as well as decelerated from 275 to $260 \mathrm{~m} / \mathrm{s}$ using an array of dipole lenses in AG configuration.

A scheme of the experimental setup is shown in Fig. 1. The prototype AG decelerator consists of 12 dipole lenses, positioned symmetrically around the molecular beam axis. One of the lenses is shown enlarged in the inset at the left-hand side of Fig. 1, along with a $(\hat{x}, \hat{y}, \hat{z})$-axis system. A lens is composed of two identical thin electrodes $(2 \mathrm{~mm}$ thick) with the side facing the molecular beam rounded off with a radius of curvature of $1 \mathrm{~mm}$. The distance between 
the two electrodes is $2 \mathrm{~mm}$. Typically, voltages of +10 and $-10 \mathrm{kV}$ are applied to these electrodes, yielding an electric field at the molecular beam axis close to $90 \mathrm{kV} / \mathrm{cm}$. The electric fields along $\hat{x}$ and $\hat{y}$ are shown in the inset at the right-hand side of Fig. 1. It is seen that molecules in high-field seeking states experience a force that repels them from the molecular beam axis in the plane of the electrodes, and attracts them toward the molecular beam axis in the plane perpendicular to this. The force acting on a polar molecule is $\vec{F}=-\vec{\nabla} W(E)$, with $W(E)$ being the Stark energy of the molecule in the electric field of magnitude $E$. Based on symmetry arguments, $\vec{\nabla} \cdot \vec{F}=0$ on the molecular beam axis for a molecule with a first or second order Stark interaction [11]. Assuming the electrodes to be infinitely long, $F_{z}$ is equal to zero, and it follows that $\partial F_{x} / \partial x=-\partial F_{y} / \partial y$.

Close to the molecular beam axis the force is linear to the displacement. For molecules moving with a constant velocity $v_{z}$ along the molecular beam axis, the equations of motion along $\hat{x}$ and $\hat{y}$ can therefore be written as $\partial^{2} x / \partial z^{2}+\Omega^{2} x=0$ and $\partial^{2} y / \partial z^{2}-\Omega^{2} y=0$ with $\Omega=\sqrt{\left|k_{x, y}\right| / m v_{z}^{2}}$ and $k_{x}$ and $k_{y}$ the force constants. Following standard procedures in ion optics $[10,17]$ the general solution in $\hat{x}$ and $\hat{y}$ can be written in matrix form as

$$
\begin{aligned}
& \left(\begin{array}{c}
x \\
v_{x}
\end{array}\right)=\left(\begin{array}{cc}
\cos \Omega L & {\left[1 /\left(v_{z} \Omega\right)\right] \sin \Omega L} \\
-v_{z} \Omega \sin \Omega L & \cos \Omega L
\end{array}\right)\left(\begin{array}{c}
x_{0} \\
v_{x 0}
\end{array}\right) \equiv F\left(\begin{array}{c}
x_{0} \\
v_{x 0}
\end{array}\right), \\
& \left(\begin{array}{c}
y \\
v_{y}
\end{array}\right)=\left(\begin{array}{cc}
\cosh \Omega L & {\left[1 /\left(v_{z} \Omega\right)\right] \sinh \Omega L} \\
v_{z} \Omega \sinh \Omega L & \cosh \Omega L
\end{array}\right)\left(\begin{array}{c}
y_{0} \\
v_{y 0}
\end{array}\right) \equiv D\left(\begin{array}{c}
y_{0} \\
v_{y 0}
\end{array}\right),
\end{aligned}
$$

where the $2 \times 2$ matrices describe the transfer of the molecules through a focusing $(F)$ and defocusing $(D)$ lens of length $L$. The path of a molecule through the array of lenses can now be calculated, realizing that the molecules pass alternatingly through focusing and defocusing lenses. The field-free regions of length $s$ in between the lenses are described by a simple transfer matrix $O$ of the form

$$
O=\left(\begin{array}{cc}
1 & s / v_{z} \\
0 & 1
\end{array}\right)
$$

The transfer matrix of the complete AG decelerator with $2 N$ lenses is given by $(F O D O)^{N}$. In order for the transverse motion to be stable it is necessary that
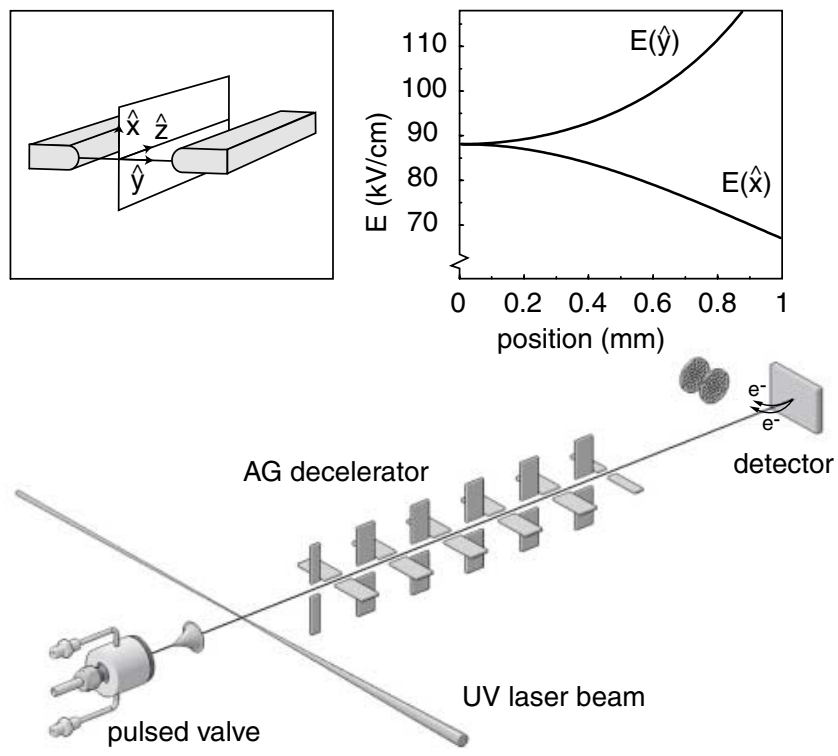

FIG. 1. Scheme of the experimental setup. In the inset at the left-hand side of the figure an enlarged view of a lens is shown together with a $(\hat{x}, \hat{y}, \hat{z})$-axis system. The electric fields along $\hat{x}$ and $\hat{y}$ are shown in the inset at the right-hand side of the figure. all the elements of this transfer matrix remain bounded when $N$ increases indefinitely, which is the case when $-1<\frac{1}{2} \operatorname{Tr}(F O D O)<+1$.

In Fig. 2 typical trajectories through the decelerator are shown using experimental parameters. The gray-shaded area indicates the region of stable trajectories. It is seen that the beam envelope is larger at the positive lenses than at the negative lenses.

In order to decelerate or accelerate the molecules, timevarying electric fields are applied. A molecule in a highfield seeking state will gain kinetic energy as it enters the field of a lens, while it loses kinetic energy as it leaves the lens. When the electric field is switched on when the molecule is inside a lens, there will be no change to its kinetic energy but it will decelerate while leaving the lens. The moment the field is switched on determines the effective length $L$ of the lens; the focusing properties
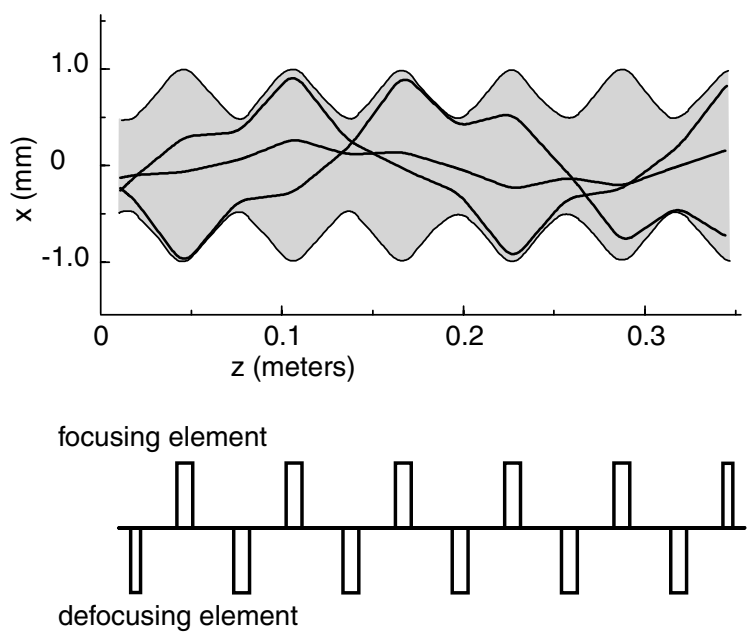

FIG. 2. A number of trajectories through the AG decelerator, calculated using the matrix formalism described in the text, are shown. The gray-shaded area shows the beam envelope. 
can thus be optimized without affecting the deceleration properties. Phase stable operation [7] can be obtained by switching the electric fields off again when the molecules have not yet left the field of a lens completely. In that case, molecules which are at the head of the pulse will lose more kinetic energy while molecules which are at the pulse's tail will lose less kinetic energy. Therefore, molecules in a narrow position and velocity interval will be confined to this area of phase space throughout the decelerator (see Fig. 3). It is convenient to define a phase angle $(\phi)$ as a measure of the energy loss per stage $(\Delta W)$ via $\Delta W=$ $W^{\prime} \sin \phi$, with $W^{\prime}$ the maximum (negative) Stark shift on the molecular beam axis.

In order to demonstrate the performance of the alternate gradient decelerator, experiments have been carried out on $\mathrm{CO}$ molecules in the $a^{3} \Pi$ state. The main reason for choosing metastable $\mathrm{CO}$ molecules is that (i) they can be prepared in a single quantum state at a well-defined position and time, and (ii) their velocity distribution can be readily recorded.

A pulsed beam of $\mathrm{CO}$ is produced by expanding a $5 \%$ $\mathrm{CO}$ in $\mathrm{Xe}$ mixture into vacuum, using a modified solenoid valve. Cooling the valve housing to $180 \mathrm{~K}$ (just above the boiling point of $\mathrm{Xe}$ ) reduces the mean velocity of the $\mathrm{CO}$ molecules in the beam to $275 \mathrm{~m} / \mathrm{s}$, corresponding to an initial kinetic energy of $E_{\text {kin }}=89 \mathrm{~cm}^{-1}$. The velocity

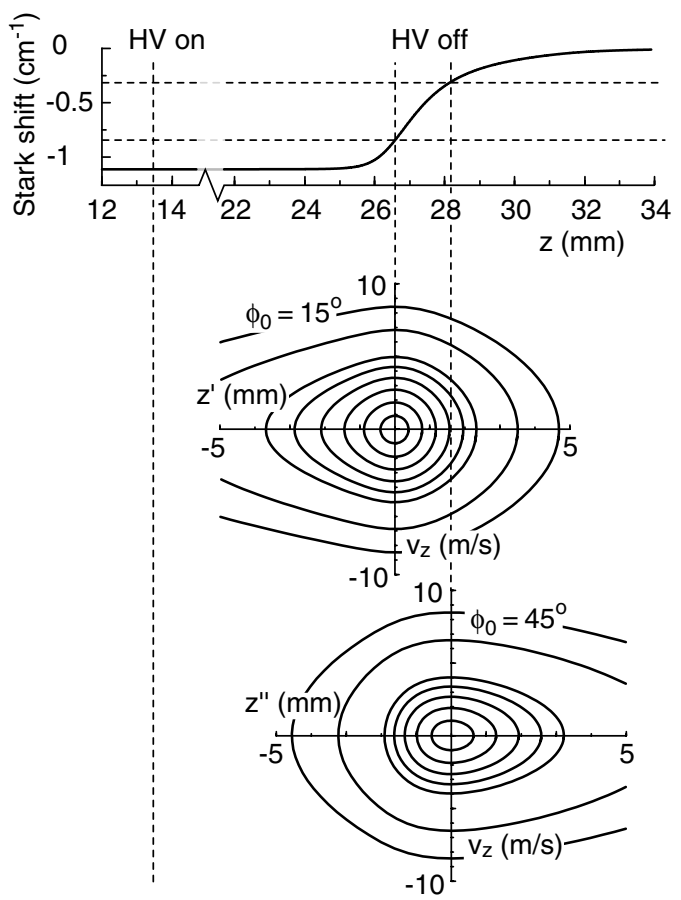

FIG. 3. The Stark shift of metastable $\mathrm{CO}\left(a^{3} \Pi_{1}, v^{\prime}=0, J^{\prime}=\right.$ $1, M \Omega=1)$ molecules as a function of their position along the molecular beam axis. The dotted lines indicate the position of the synchronous molecule when the electric fields are switched on and off for two different phases. In the lower part of the figure some (closed) trajectories in phase space are shown for nonsynchronous molecules, relative to the position and velocity of the synchronous molecule. spread is approximately $10 \%$, corresponding to a temperature of $\sim 0.5 \mathrm{~K}$ in the moving frame. The $\mathrm{CO}$ molecules pass through a 1.0-mm-diam skimmer into a second, differentially pumped, vacuum chamber. Metastable $\mathrm{CO}$ molecules in a single quantum state are prepared by direct laser excitation of the ground state molecules on the spin-forbidden $a^{3} \Pi\left(v^{\prime}=0\right) \leftarrow X^{1} \Sigma^{+}\left(v^{\prime \prime}=0\right)$ transition, using pulsed $206 \mathrm{~nm}(6.0 \mathrm{eV})$ radiation. For this experiment the molecules need to be prepared in states that experience a negative Stark shift which are the lower components of the $\Lambda$ doublets in the $a^{3} \Pi$ state [18]. In the experiments reported here, laser preparation of the $J^{\prime}=1 a^{3} \Pi_{1}$ level via the $R_{2}(0)$ transition is used. By setting the polarization of the laser perpendicular to the stray fields present in the decelerator only the $M \Omega=1$ high-field seeking state is excited.

Laser preparation is performed in a 1-mm-diam spot, $5 \mathrm{~mm}$ in front of the $35-\mathrm{cm}$-long AG decelerator consisting of an array of 12 equidistant 27-mm-long dipole lenses. Successive lenses are separated by a 4-mm-long drift region. The two opposing electrodes of a lens are simultaneously switched by two independent high voltage switches to maximum voltages of +10 and $-10 \mathrm{kV}$. The first and last lenses have just half the length in order to have a more symmetric acceptance along $\hat{x}$ and $\hat{y}$. The time-of-flight (TOF) distributions over the $54 \mathrm{~cm}$ distance from laser preparation to detection are recorded by measuring the number of electrons emitted from a flat gold surface when the metastable $\mathrm{CO}$ molecules impinge on it.

In Fig. 4 the measured TOF distributions are plotted for several values of $\phi_{0}$, as indicated. The lower curve is the TOF distribution of the original beam, when no voltages are applied, corresponding to a beam with a mean velocity of $275 \mathrm{~m} / \mathrm{s}$. Using the 12 stages, molecules are decelerated from 275 to $260 \mathrm{~m} / \mathrm{s}$, or accelerated from 275 up to $289 \mathrm{~m} / \mathrm{s}$, depending on the phase angle $\phi_{0}$ that is used. The thin curves show the results of a Monte Carlo simulation using a finite element method to calculate the electric fields. The calculations are seen to describe the TOF distributions for the decelerated bunch rather well; similarly, good agreement is obtained in the simulation for the accelerated beam (not shown). In order to match the measured TOF distributions we have scaled down the simulations by a factor of 20; upon applying the electric fields the integrated signal decreases by a factor of $\sim 7$, where we would have expected an increase in signal by at least a factor of 3. This discrepancy is most likely due to misalignments in the decelerator; with a random displacement of $\pm 0.2 \mathrm{~mm}$ of the lenses, the observed TOF distributions can be quantitatively reproduced.

The proof-of-principle experiment described in this Letter demonstrates the potential of an AG decelerator for producing beams of polar molecules with arbitrarily (low) velocities. In the deceleration process the phase-space density remains constant; bunches of molecules are kept together independent of the number of lenses used in the 


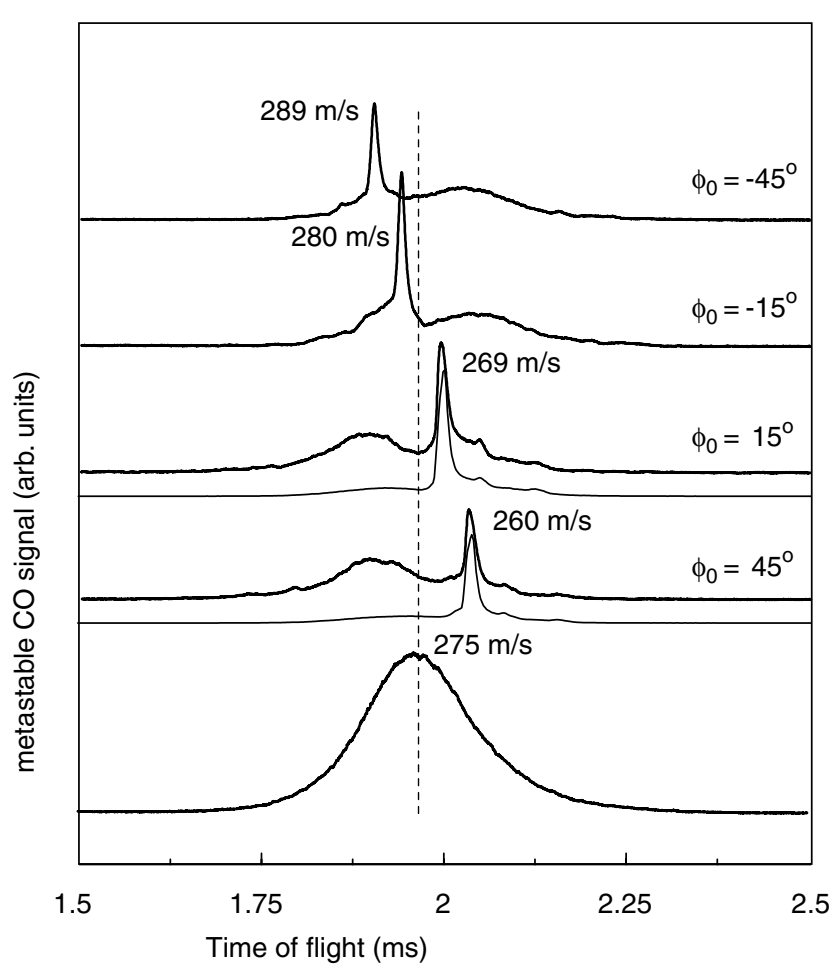

FIG. 4. Observed TOF distributions of metastable CO over the $54 \mathrm{~cm}$ path length through the apparatus for four different phases as indicated in the figure, together with the TOF distribution when no electric fields are applied (lower curve). The measurements (thick curves) have been given an offset for clarity. The thin curves show the results of a Monte Carlo simulation. The dashed line shows the expected arrival time of a molecule flying with a (constant) velocity of $275 \mathrm{~m} / \mathrm{s}$.

decelerator. With the present geometry, 90 lenses would be required to bring the metastable $\mathrm{CO}$ molecules to a total standstill. For this, the mechanical design of the decelerator must be improved. Alternatively, a geometry where a single lens is composed of multiple deceleration stages can be used, i.e., a geometry with a number of subsequent electrode pairs oriented along the same plane. The latter design is expected to be less critical to misalignments. Once sufficiently slow (below, say, $25 \mathrm{~m} / \mathrm{s}$ ) the molecules can be trapped in an electrodynamic trap $[19,20]$ or in a ring geometry, for instance, around a wire $[18,21]$ or in an AG storage ring [11,22]. The AG deceleration and trapping scheme is applicable to samples of molecules in their lowest energy quantum state, which are stable against losses via inelastic collisions [23]. Therefore, a further increase of phase-space density via evaporative cooling might be feasible. The AG deceleration scheme is suited for decelerating not only small polar molecules but also relatively heavy molecules with large dipole moments and small rotational constants. In particular, the AG decelerator can be used to slow down molecules that are used in studies aimed at testing the time reversal symmetry [24] and at testing parity violation induced by the weak interaction [25]. The increased interaction time when using slow molecules will be highly beneficial for the attainable spectral resolution in these studies.

This work is part of the research program of the "Stichting voor Fundamenteel Onderzoek der Materie (FOM)," which is financially supported by the "Nederlandse Organisatie voor Wetenschappelijk Onderzoek (NWO)." The research of R. T. J. has been made possible by the Royal Netherlands Academy of Arts and Sciences. We acknowledge the help of Dr. Giel Berden with the experiments.

[1] J. M. Doyle and B. Friedrich, Nature (London) 401, 749 (1999).

[2] C. J. Williams and P. S. Julienne, Science 287, 986 (2000).

[3] M. Baranov, L. Dobrek, K. Góral, L. Santos, and M. Lewenstein, cond-mat/0201100.

[4] H. L. Bethlem et al., Nature (London) 406, 491 (2000).

[5] F. M. H. Crompvoets, H. L. Bethlem, R. T. Jongma, and G. Meijer, Nature (London) 411, 174 (2001).

[6] H. L. Bethlem, G. Berden, and G. Meijer, Phys. Rev. Lett. 83, 1558 (1999).

[7] H. L. Bethlem, G. Berden, A. J. A. van Roij, F. M. H. Crompvoets, and G. Meijer, Phys. Rev. Lett. 84, 5744 (2000).

[8] J. A. Maddi, T. P. Dinneen, and H. Gould, Phys. Rev. A 60, 3882 (1999).

[9] W. H. Wing, Prog. Quantum Electron. 8, 181 (1984).

[10] E. D. Courant and H. S. Snyder, Ann. Phys. (N.Y.) 3, 1 (1958).

[11] D. Auerbach, E. E. A. Bromberg, and L. Wharton, J. Chem. Phys. 45, 2160 (1966).

[12] R. Wolfgang, Sci. Am. 219, No. 4, 44 (1968).

[13] D. Kakati and D. C. Lainé, Phys. Lett. 24A, 676 (1967); 28A, 786 (1969); J. Phys. E 4, 269 (1971).

[14] F. Günther and K. Schügerl, Z. Phys. Chem. Neue Folge 80, 155 (1972); A. Lübbert, F. Günther, and K. Schügerl, Chem. Phys. Lett. 35, 210 (1975); A. Lübbert, G. Rotzoll, and F. Günther, J. Chem. Phys. 69, 5174 (1978).

[15] E. E. A. Bromberg, Ph.D. thesis, University of Chicago, 1972.

[16] H-R. Noh, K. Shimizu, and F. Shimizu, Phys. Rev. A 61, 041601(R) (2000).

[17] S. Y. Lee, Accelerator Physics (World Scientific, Singapore, 1999).

[18] R. T. Jongma, G. von Helden, G. Berden, and G. Meijer, Chem. Phys. Lett. 270, 304 (1997).

[19] F. Shimizu and M. Morinaga, Jpn. J. Appl. Phys. 31, L1721 (1992).

[20] E. Peik, Eur. Phys. J. D 6, 179 (1999).

[21] H. J. Loesch and B. Scheel, Phys. Rev. Lett. 85, 2709 (2000).

[22] H. Gould, in Proceedings of the U.S. DOE BES 2000 Meeting of the AMOP Program, Book of Abstracts (to be published).

[23] J. L. Bohn, Phys. Rev. A 63, 052714 (2001).

[24] E. A. Hinds, Phys. Scr. T70, 34 (1997).

[25] Ch. Daussy et al., Phys. Rev. Lett. 83, 1554 (1999). 